\title{
Saccadic Eye Velocity after Selective GABAergic Treatment with Tiagabine in Healthy Volunteers
}

\author{
Peter Zwanzger ${ }^{a}$ Cornelius Schüle ${ }^{a}$ Daniela Eser ${ }^{a}$ Thomas C. Baghai \\ Frank Padberg $^{a}$ Robin Ella ${ }^{a}$ Hans-Jürgen Möller ${ }^{a}$ Rainer Rupprecht ${ }^{a}$ \\ Norbert Kathmann ${ }^{b}$ \\ ${ }^{a}$ Department of Psychiatry, University of Munich, Munich; ${ }^{b}$ Department of Psychology, Humboldt-University, \\ Berlin, Germany
}

\section{Key Words}

Saccadic eye velocity $\cdot \gamma$-Aminobutyric acid $\cdot$ Tiagabine fects or the different site of action on the $G_{A B A} / B Z D$ receptor complex may account for this deviating profile.

Copyright $(2005$ S. Karger AG, Basel

\section{Introduction}

Saccadic eye movements are controlled by frontal eye fields, substantia nigra, superior colliculus, pontine reticular formation and cerebellum. These regions contain high amounts of $\mathrm{GABA}_{\mathrm{A}}$ receptors [1]. Saccade eye velocity (SEV) is mediated by the superior colliculus, which is under tonic GABAergic control. Studies in primates have shown that injection of GABA agonists into the superior colliculus induce a selective reduction in SEV whereas injection of a GABA antagonist leaves SEV unchanged [2]. Hommer et al. [3] showed that intravenous administration of benzodiazepines leads to a decrease of SEV in a dose-dependent manner, accompanied by a decrease in plasma cortisol levels and an increase in self-rated sedation and plasma growth hormone. They therefore suggested SEV as a quantifiable and reliable parameter to evaluate changes in sensitivity of the $\mathrm{GABA}_{\mathrm{A}} / \mathrm{BZD}$ receptor in humans. Moreover, SEV has also been used as an

\section{KARGER \\ Fax +4161306 1234 E-Mail karger@karger.ch} www.karger.com
(C) 2005 S. Karger AG, Basel 0302-282X/05/0523-0147\$22.00/0

Accessible online at: www.karger.com/nps
Peter Zwanzger, MD

Department of Psychiatry, University of Munich

Nussbaumstrasse 7

DE-80336 Munich (Germany)

Tel. +49 895160 5813, Fax +49 895160 5869, E-Mail zwanzger@med.uni-muenchen.de 
Table 1. SEV (degrees/s), saccade amplitude (degrees), and saccade latency (ms) for $7^{\circ}, 14^{\circ}$, and $21^{\circ}$ amplitudes at baseline (pre) and after 7 days of tiagabine treatment (post) in a daily dose of $15 \mathrm{mg}$

\begin{tabular}{|c|c|c|c|c|c|c|}
\hline \multirow{2}{*}{$\begin{array}{l}\text { Target } \\
\text { amplitude }\end{array}$} & \multicolumn{2}{|l|}{ SEV } & \multicolumn{2}{|c|}{ Saccade amplitude } & \multicolumn{2}{|c|}{ Saccade latency } \\
\hline & pre & post & pre & post & pre & post \\
\hline $7^{\circ}$ & 187 (19) & $191(17)$ & $6.6(0.3)$ & $6.5(0.4)$ & $154(18)$ & $159(15)$ \\
\hline $14^{\circ}$ & $265(23)$ & $285(28)$ & $12.9(0.6)$ & $12.9(0.6)$ & $161(8)$ & $161(11)$ \\
\hline $21^{\circ}$ & $322(26)$ & $321(40)$ & $18.8(0.9)$ & $18.9(1.1)$ & $167(10)$ & $167(11)$ \\
\hline
\end{tabular}

Group means and standard deviation (in parentheses) are indicated. objective measure of sedation [4]. Investigations in patients with panic disorder have shown that these patients show a reduced sensitivity of the $\mathrm{GABA}_{\mathrm{A}} / \mathrm{BZD}$ receptor to benzodiazepines compared with healthy controls [5]. In patients with premenstrual dysphoric disorder a decreased SEV appears to reflect pathophysiological alterations implicated in this disorder [6].

Tiagabine is a new antiepileptic compound that exerts its anticonvulsant effects via blockade of the GABA transporter I. Tiagabine inhibits GABA reuptake from the synaptic cleft and consequently leads to an increase in GABAergic neurotransmission [7]. Apart from anticonvulsant properties also anxiolytic activity has been noted in patients with panic disorder [8].

To further characterize the impact of tiagabine on $\mathrm{GABA}_{\mathrm{A}}$ receptor function in humans we investigated the effects of tiagabine on SEV assuming that this measure may be indicative of $\mathrm{GABA}_{\mathrm{A}} / \mathrm{BZD}$ receptor sensitivity. We hypothesized that treatment with tiagabine might decrease SEV in healthy subjects.

\section{Methods}

Ten healthy volunteers ( 7 males, 3 females, mean age $27.6 \pm 4$ years) were investigated. They were screened for a history of mental or somatic diseases. None of the participants had taken opiates or benzodiazepines during 4 weeks before the experimental session, as assessed by urine toxicology screening. The protocol was approved by the local ethics committee of the Medical Department. All subjects had given their written informed consent after a complete description of the study.

At baseline and after 7 days of tiagabine treatment saccadic velocity was tested at 9.00 o'clock a.m. Tiagabine was administered during 7 consecutive days 3 times daily in a daily dose of $15 \mathrm{mg}$. To check the subjects' compliance to drug intake serum levels of tiagabine were determined by high-performance liquid chromatography. For the oculomotor test subjects performed a saccade task which required them to follow a white target dot (diameter: $3 \mathrm{~mm}$ ) on a dark background of a 17-inch computer monitor as quickly and accurately as possible. The distance between the eyes and the monitor was $28 \mathrm{~cm}$. Starting from a central fixation position the dot randomly jumped to $7^{\circ}, 14^{\circ}$, or $21^{\circ}$ right or left of the center, remained there for 1,200-2,000 $\mathrm{ms}$ (random variation) and then returned to the center. After a variable period between 1,200 and $2,000 \mathrm{~ms}$ the next target step started. 240 test trials were presented after completion of 10 familiarization trials. Generation and timing of the visual target was software-controlled (ERTS, BeriSoft Corp.). Eye movements were recorded using an infrared pupil-cornea reflection system (Eye Trac 210, ASL Corp.) and stored (sampling frequency: $500 \mathrm{~Hz}$ ) together with event markers on a personal computer for offline analysis. Head movement was strictly discouraged by instruction and minimized by a chin and forehead rest. We estimated SEV as peak velocity of individual saccades by computing mean velocity and multiplying this value by 1.64 [1]. Mean velocity was computed by dividing the measured amplitude of each saccade by the duration of the respective saccade. Start and end point of a saccade were determined by hand and rechecked by an independent rater. In addition, amplitudes and latencies of saccades were assessed. Only correct saccades, i.e. saccades where the subject fixated the central dot during a 400-ms baseline and when the saccade amplitude showed a gain between 0.7 and 1.1 were used in the analyses. For each participant median peak velocities, amplitudes, and latencies were then computed separately for each target amplitude in the pre- and post-treatment sessions. These values were entered into ANOVAs for repeated measures with Target Amplitude $\left(7^{\circ}, 14^{\circ}, 21^{\circ}\right)$ and Treatment (pre- vs. post-) as factors; $p<0.05$ was used as the level of significance. Greenhouse-Geisser corrections were applied where necessary.

\section{Results}

Eight out of 10 subjects remained in the analysis (6 male, 2 female, mean age $28 \pm 4$ years). Two subjects were excluded due to technical artifacts. Descriptive results are shown in table 1 . There was a significant increase in SEV with increasing amplitude [main effect of target amplitude: $\mathrm{F}(2,14)=155.0, \varepsilon=0.65, \mathrm{p}<0.001]$. The effect of tiagabine Treatment (pre- vs. post-) on SEV showed a nonsignificant trend for higher SEV after treatment $[F(1,7)=4.26, p=0.078]$. There was also a trend for an interaction of Target Amplitude $\times$ Treatment $[\mathrm{F}(2$, 14) $=3.39, \varepsilon=0.72, p=0.085]$. Paired comparisons revealed that only with $14^{\circ}$ targets SEV increased after ti- 
agabine $[\mathrm{t}(7)=-3.95, \mathrm{p}=0.006]$. To test whether SEV is affected by varying saccade amplitudes across time, ANOVA was also performed for saccade amplitudes. No effect was found for treatment on saccade amplitudes $[F(1,7)=0.01, p=0.93]$ nor was there an interaction of Treatment $\times$ Target Amplitude $[\mathrm{F}(2,14)=0.16, \varepsilon=0.68$, $\mathrm{p}=0.77]$. Saccade latencies showed a significant effect of Target Amplitude $[\mathrm{F}(2,14)=6.77, \varepsilon=0.65, \mathrm{p}=0.023]$, indicating increasing latencies with increasing eccentricity of targets. Treatment had a marginal effect $[\mathrm{F}(1,7)=$ $5.36, p=0.054]$, which is due to a trend for delayed saccade onset with $7^{\circ}$ targets after tiagabine treatment $[\mathrm{t}(7)=-2.27, \mathrm{p}=0.057]$.

Apart from slight vertigo, dizziness and slight sedation in the first 3 days of treatment tiagabine was safe and well tolerated by all subjects. None reported any sedation or loss of concentration after the treatment period. All subjects reached sufficient tiagabine plasma levels (mean 126 $\pm 11 \mathrm{ng} / \mathrm{ml}$ ).

\section{Discussion}

In conclusion, we found no effects of treatment with the GABA reuptake blocker tiagabine on SEV. After a 7day period of treatment with tiagabine $15 \mathrm{mg}$ daily, peak SEV remained unchanged. This is in contrast to prior findings with benzodiazepines administered to experimental animals and healthy subjects. Hikosaka and Wurtz [2] found a marked reduction of SEV after injection of a GABA agonist in the superior colliculus, which is supposed to control SEV. Moreover, studies in healthy subjects showed a reduction of SEV after injection of diazepam [3] or midazolam [9].

However, it has to be considered that these studies investigated changes in SEV only after intravenous injection of benzodiazepines. In addition, only short-term effects were assessed. Ball et al. [10] observed that effects of repeated midazolam injections on SEV showed a relatively fast return to baseline within 15-30 min and suggested that this either indicates an acute tolerance to effects of benzodiazepines or reflects a redistribution phenomenon. In view of the rapid changes and the absence of midazolam metabolites this phenomenon could not be explained with benzodiazepine metabolism. In the light of these findings, the subchronic administration or tolerance effects might explain the lack of influence of tiagabine treatment on SEV in our study. However, van Steveninck et al. [11] suggested that saccadic eye movement parameters reliably reflect plasma benzodiazepine levels.
As all subjects in our sample reached sufficient tiagabine plasma levels, bioavailability of the compound cannot account for our findings.

Moreover, studies investigating the effects of benzodiazepine injection after chronic benzodiazepine administration in patients with panic disorder showed a striking reduction of benzodiazepine receptor sensitivity $[9,12]$ reflected by an attenuated reduction of SEV after intravenously administered benzodiazepines. One hypothesis is a possible decrease in the number of benzodiazepine receptors after long-term treatment with benzodiazepines [9]. Therefore, differences in $\mathrm{GABA}_{\mathrm{A}} / \mathrm{BZD}$ receptor density following benzodiazepine or tiagabine administration might also be responsible for the lack of change of SEV after tiagabine treatment [9].

However, to our knowledge there is no study investigating the effects of drugs targeting selectively the GABA binding site of the $\mathrm{GABA}_{\mathrm{A}} / \mathrm{BZD}$ receptor complex on SEV in humans so far. Interestingly, sleep studies showed that agonists for the GABA binding site produce a much more favorable sleep EEG profile compared to benzodiazepines with no REM suppression, an enhancement of slow wave activity during non-REM sleep, and no withdrawal effects [13]. Given the fact that there is no report of dependency or withdrawal after tiagabine treatment so far, it seems possible that there exists a different effect profile of tiagabine on GABA receptor function, and this might be reflected by SEV measures like those presented here.

Since tiagabine treatment did not affect SEV and since there was only a trend for changes in saccadic latency as measures of sedation and attention [4], advantages of selective GABAergic treatment with tiagabine compared to benzodiazepines can be suggested.

Neuropsychobiology 2005;52:147-150 


\section{References}

1 Becker W: Metrics; in Wurtz RH, Goldberg ME (eds): The Neurobiology of Saccadic Eye Movements. Amsterdam, Elsevier Science, 1989.

2 Hikosaka O, Wurtz RH: Modification of saccadic eye movements by GABA-related substances. I. Effect of muscimol and bicuculline in monkey superior colliculus. J Neurophysiol 1985;53:266-291.

3 Hommer DW, Matsuo V, Wolkowitz O, Chrousos G, Greenblatt DJ, Weingartner H, Paul SM: Benzodiazepine sensitivity in normal human subjects. Arch Gen Psychiatry 1986; 43:542-551.

4 Gao F, Mapleson WW, Vickers MD: Effect of sub-anaesthetic infusions of propofol on peak velocity of saccadic eye movements. Eur J Anaesthesiol 1991;8:267-276.
5 Roy-Byrne PP, Cowley DS, Greenblatt DJ, Shader RI, Hommer D: Reduced benzodiazepine sensitivity in panic disorder. Arch Gen Psychiatry 1990;47:534-538.

6 Sundstrom I, Backstrom T: Patients with premenstrual syndrome have decreased saccadic eye velocity compared to control subjects. Biol Psychiatry 1998;44:755-764.

7 Meldrum BS, Chapman AG: Basic mechanisms of gabitril (tiagabine) and future potential developments. Epilepsia 1999;40(suppl 9): S2-6.

8 Zwanzger P, Baghai T, Schüle C, Minov C, Padberg F, Möller HJ, Rupprecht R: Tiagabine improves panic and agoraphobia in panic disorder patients. J Clin Psychiatry 2001;62:656657.

9 Potokar J, Coupland N, Wilson S, Rich A, Nutt D: Assessment of GABA(A)benzodiazepine receptor $(\mathrm{GBzR})$ sensitivity in patients on benzodiazepines. Psychopharmacology (Berl) 1999;146:180-184.
10 Ball DM, Glue P, Wilson S, Nutt DJ: Pharmacology of saccadic eye movements in man. 1. Effects of the benzodiazepine receptor ligands midazolam and flumazenil. Psychopharmacology (Berl) 1991;105:361-367.

11 van Steveninck AL, Schoemaker HC, Pieters MS, Kroon R, Breimer DD, Cohen AF: A comparison of the sensitivities of adaptive tracking, eye movement analysis and visual analog lines to the effects of incremental doses of temazepam in healthy volunteers. Clin Pharmacol Ther 1991;50:172-180.

12 Cowley DS, Roy-Byrne PP, Radant A, Ritchie JC, Greenblatt DJ, Nemeroff CB, Hommer DW: Benzodiazepine sensitivity in panic disorder: effects of chronic alprazolam treatment. Neuropsychopharmacology $1995 ; 12$ : 147157.

13 Lancel M, Faulhaber J, Deisz RA: Effect of the GABA uptake inhibitor tiagabine on sleep and EEG power spectra in the rat. Br J Pharmacol 1998; 123:1471-1477. 